\title{
Uso de células de carga para mensuração da força dos membros inferiores em nado ondulatório
}

\author{
Marcelo Papoti 1,3 \\ Ricardo Vitório 2 \\ André B. Velosa ${ }^{3}$ \\ Sergio A. Cunha 4 \\ Adelino S. Ramos da Silva ${ }^{3}$ \\ Luiz E. B. Martins 5 \\ Claudio A. Gobatto ${ }^{3}$
}

https://doi.org/10.5628/rpcd.07.03.313

\section{RESUMO}

Considerando a crescente utilização da técnica de nado ondulatório submerso $\left(\mathrm{NO}_{\mathrm{SB}}\right)$ durante a saída e as viradas nas provas de nado crawl, o presente estudo objetivou determinar a força dos membros inferiores em nado ondulatório $\left(\mathrm{FMI}_{\mathrm{NO}}\right)$ e suas correlações com as performances máximas do $\mathrm{NO}_{\mathrm{SB}}$ e na superfície $\left(\mathrm{NO}_{\mathrm{SP}}\right)$. Para isso 19 nadadores com idade de $15,2 \pm 0,6$ anos filiados a federação aquática paulista foram submetidos a um esforço máximo de 30 s de $\mathrm{FMI}_{\mathrm{NO}}$, atados a um dinamômetro contendo células de carga como elemento sensor primário. A tensão detectada pelo dinamômetro, devido aos esforços dos nadadores, foi amplificada por uma fonte de extensometria. Os valores obtidos foram enviados ao computador armazenados na freqüência de $200 \mathrm{~Hz}$ e suavizados utilizando o filtro "butterworth" de quarta ordem com freqüência de corte de $3 \mathrm{~Hz}$. Com a utilização da reta de calibração podese converter valores de tensão $(\mathrm{mV})$ em unidades de força $(\mathrm{N})$ pelo programa Matlab 5.3, possibilitando assim a determinação dos valores médios de $\mathrm{FMI}_{\mathrm{NO}}$. Posteriormente os nadadores realizaram esforços máximos de $15 \mathrm{~m}$ de $\mathrm{NO}_{\mathrm{SB}}$ e de $25 \mathrm{~m}$ $\mathrm{NO}_{\mathrm{sp}}$. As possíveis relações entre esses três esforços, foram analisadas utilizando o teste de correlação de Pearson com nível de significância pré-fixado para $\mathrm{P}<0,05$. Verificamos valores de $53,85 \pm 11,45 \mathrm{~N}, 1,19 \pm 0,14 \mathrm{~m} . \mathrm{s}^{-1}$ e $1,76 \pm 0,26 \mathrm{~m} . \mathrm{s}^{-1}$ para a $\mathrm{FMI}_{\mathrm{NO}}, \mathrm{NO}_{\mathrm{SB}}$ e $\mathrm{NO}_{\mathrm{SP}}$ respectivamente. $\mathrm{A} \mathrm{FMI}_{\mathrm{NO}}$ apresentou significativas correlações com a NO $\mathrm{NB}_{\mathrm{SB}}(\mathrm{r}=0,80)$ e $\mathrm{NO}_{\mathrm{SP}}$ $(r=0,76)$. Os resultados do presente estudo sugerem a utiliza-

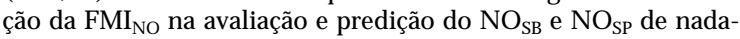
dores treinados. No entanto mais pesquisas são necessárias para verificar a sensibilidade da $\mathrm{FMI}_{\mathrm{NO}}$ aos efeitos específicos do treinamento em natação.

Palavras-chave: nado ondulatório; nado atado; mensuração de força.

\author{
1 Faculdades Integradas de Bauru - CEPA F, Brasil \\ 2 Laboratório de Pesquisa em Educação Física - LAPEF \\ UNESP, Brasil \\ 3 Laboratório de Fisiologia A plicada ao Esporte, IB \\ UNESP, Brasil \\ ${ }^{4}$ Laboratório de A nálises Biomecânicas, UNESP, Brasil \\ 5 Laboratório para instrumentação em Fisiologia \\ do Exercício, UNICAM P, Brasil
}

\section{ABSTRACT \\ Use of load cells to measurements of underwater dolphin kicki force in swimming tethered}

Considering the crescent utilization of the underwater dolphin kick $\left(\mathrm{NO}_{S B}\right)$ techniques during the beginning and the turns of front crawl events, the main purpose of the present study was to determine the dolphin kick force $\left(F M I_{N O}\right)$ in tethered swimming and its correlations with the maximal performance determined in $\mathrm{NO}_{S B}$ and in dolphin kick with a commercial board $\left(\mathrm{NO}_{\mathrm{SP}}\right)$. Nineteen male swimmers with mean age of $15.2 \pm 0.6$ years and affiliated with Sao Paulo A quatic Federation participated in the present study. The athletes were submitted a 30-s maximal effort of dolphin kick tethered to a dynamometer with strain gages (load cells) as a primary sensor element. The tension detected by the dynamometer due the swimmers' efforts was amplified by an extensometer font. The results obtained during the efforts were sent to a computer using an interface and were stored in a data acquisition program at $200 \mathrm{~Hz}$. After these procedures, the results were smoothed using the "butterworth" filter of fourth order with $3-\mathrm{Hz}$ fre quency. Using the calibration straight line, the values were converted into force units $(\mathrm{N}$ ) by the $\mathrm{M}$ atlab 5.3 program. This conversion allowed the determination of the dolphin kick mean force in tethered swimming $\left(\mathrm{FMI}_{\mathrm{NO}}\right)$. Then, the swimmers performed a $15-\mathrm{m}$ maximum effort of underwater dolphin kick $\left(\mathrm{NO}_{S B}\right)$ and another $25-\mathrm{m}$ maximum effort of dolphin kick using a commercial board $\left(\mathrm{NO}_{\mathrm{SP}}\right)$. The relationships between these three maximum efforts were analyzed using the Pearson's correlation coefficient. A significance level of $5 \%$ was chosen. The mean \pm standard deviation of $\mathrm{FMI}_{\mathrm{NO}}, \mathrm{NO}_{S B}$ and $\mathrm{NO}_{S P}$ were $53.85 \pm 11.45 \mathrm{~N}, 1.19 \pm 0.14 \mathrm{~m} . \mathrm{s}^{-1}$, and $1.76 \pm 0.26 \mathrm{~m} . \mathrm{s}^{-1}$, respectively. The $\mathrm{FMI}_{\mathrm{NO}}$ presented significant correlations with $\mathrm{N} \mathrm{O}_{S B}(\mathrm{r}=$ $0.80)$ and $N O_{S P}(r=0.76)$. According to the results of the present study, the use of $\mathrm{FMI}_{\mathrm{NO}}$ to evaluate and predict the $\mathrm{NO}_{S B}$ and $\mathrm{NO}_{S P}$ of expert's swimmers is suggested. However, more studies are necessary to verify the sensibility of the $F M I_{N O}$ to specific effects into the swimming training.

Key-words: dolphin kick; tethered swimming; force measurement. 


\section{INTRODUÇÃO}

O nado ondulatório submerso $\left(\mathrm{NO}_{\mathrm{SB}}\right)$ em decúbito ventral (para o nado borboleta) e dorsal (para o nado costas) é um procedimento tradicionalmente realizado na saída e após as viradas em natação. Nos Jogos Olímpicos de Seoul em 1988 foram verificadas significativas reduções nos recordes mundiais no nado de costas devido à utilização do $\mathrm{NO}_{S \mathrm{~B}}{ }^{(3)}$. Apesar da distância permitida para a realização desse movimento ter sido limitada para $15 \mathrm{~m}$, o $\mathrm{NO}_{\mathrm{SB}}$ ainda é muito utilizado nos os nados borboleta e costas. Recentemente o $\mathrm{NO}_{\mathrm{SB}}$ foi incorporado na saída e viradas nas provas de nado livre(3) de modo que al guns nadadores também realizam esses movimentos na posição lateral (9).

Provavelmente em função da crescente utilização do $\mathrm{NO}_{\mathrm{SB}}$, diversos estudos investigaram esses movimentos sob o aspecto cinemático(2) bem como os possíveis benefícios da incorporação dessa técnica na performance de nado livre(3). No entanto, a literatura ainda carece de um maior numero de pesquisas empenhadas em investigar o desenvolvimento de metodologias que possibilitem de avaliação e monitoramento dos efeitos do treinamento específico do $\mathrm{NO}_{\mathrm{SB}}$.

Os testes em nado atado (TNA), particularmente os que utilizam células de carga como elemento sensor primário são metodologias alternativas que possibilitam mensurar a força do nadador com sistemas de aquisição de dados $(4,8,11,13,14,15,6,20)$ e que, por permitirem a coleta de grande número de valores em um pequeno espaço de tempo, parecem apresentar vantagem sobre o sistema de anilhas(13). Além disso, os resultados de força média (utilizando nado completo) durante esforços máximos com duração entre 10 e $30 \mathrm{~s}$ em nado atado apresentaram elevadas correlações com as performances entre 25 e 600 m durante o nado livre(11,14,15,17). Yeater et al.(20) também utilizando células de carga, constataram valores médios de $119 \pm 35$ e $138 \pm 47 \mathrm{~N}$ para a força de membros inferiores nos nados crawl e peito, respectivamente. No entanto, ainda não foram encontradas na literatura, pesquisas que mensuraram a força de $\mathrm{NO}_{\mathrm{SB}} \mathrm{em}$ nado atado.

Desse modo o propósito do presente estudo foi determinar as forças máxima e média do $\mathrm{NO}_{\mathrm{SB}} \mathrm{em}$ nado atado e suas correlações com o nado ondulatório submerso e na superfície.

\section{METODOLOGIA}

Participantes

Para isso foram avaliados 19 nadadores do sexo masculino com idade, estatura, envergadura e massa corporal de 15,2 $\pm 0,6$ anos; $176,7 \pm 6,8 \mathrm{~cm} ; 181,62 \pm 5,9$ $\mathrm{cm}$ e $66,4 \pm 5,4 \mathrm{~kg}$ respectivamente da cidade de Bauru filiados a Federação Aquática Paulista e com tempo mínimo de natação competitiva de 2 anos. A veloci dade média dos nadadores para a distância de $100 \mathrm{~m}$ nado crawl $\left(1,73 \pm 0,07 \mathrm{~m} . \mathrm{s}^{-1}\right)$ corresponde a aproximadamente $89 \%$ e $82 \%$ dos recordes brasileiro para idade e mundial respectivamente.

Previamente aos testes, os nadadores realizaram um período de aquecimento típico de aproximadamente $1000 \mathrm{~m}$ em ritmo moderado (determinado subjetivamente pelos nadadores), sendo composto de esforços utilizando os 4 estilos (borboleta, costas, peito e crawl) com predominância para o nado crawl.

Os nadadores e os técnicos foram previamente informados com relação aos procedimentos a que seriam submetidos e assinaram um termo de consentimento livre e esclarecido, aprovado pelo comitê de ética em pesquisa do Instituto de Biociências da Universidade Estadual Paulista "Júlio de Mesquita Filho" Campus de Rio Claro, autorizando a participação no estudo.

\section{Testes}

Todos os testes foram realizados em piscina aberta de $25 \mathrm{~m}$, profundidade de $180 \mathrm{~cm}$ e temperatura da água de $260 \mathrm{C}$. Os nadadores foram submetidos a três testes máximos envolvendo o nado ondulatório submerso $\left(\mathrm{NO}_{\mathrm{SB}}\right)$, na superfície $\left(\mathrm{NO}_{\mathrm{SP}}\right)$ e a força dos membros inferiores em nado ondulatório ( $\mathrm{FMI}_{\mathrm{NO}}$ ) na situação de nado atado (NA).

Determinação das velocidades de nado ondulatório submerso $\left(\mathrm{NO}_{\mathrm{SB}}\right)$ e na superfície $\left(\mathrm{NO}_{\mathrm{SP}}\right)$

$\mathrm{O} \mathrm{NO}_{\mathrm{SB}}$ consistiu na realização de um esforço máximo submerso na distância de $15 \mathrm{~m}$. Somente foram considerados os nadadores que conseguiram completar todo o percurso de modo submerso. Já o $\mathrm{NO}_{\mathrm{SP}}$ consistiu na realização de um esforço máximo de 25 $m$ utilizando uma prancha comercial. Em ambos os testes, os nadadores permaneceram próximos à borda da piscina de modo que após o sinal sonoro (apito) os nadadores iniciaram os esforços a partir de um impulso na borda da piscina. O tempo de 
nado foi mensurado com a utilização de um cronômetro manual de modo que a velocidade de nado ondulatório foi determinada pela razão entre a distância de nado e o tempo total de percurso.

Determinação da força dos membros inferiores em nado ondulatório $\left(\mathrm{FMI}_{\mathrm{NO}}\right)$

Para determinação da $\mathrm{FMI}_{\mathrm{NO}}$, os nadadores foram atados ao aparato de medição padronizado por Papoti et al.(15). Esse equipamento é constituído por um sistema de aquisição de dados tendo células de carga como elemento sensor primário suspenso sobre 2 traves de madeira de modo a permitir a calibração e mensuração da força em nado atado.

A deformação detectada pelas células de cargas (strain gage), devido à tensão gerada pelos esforços do nadador, foi amplificada por uma fonte de extensometria portátil (SODMEX ME-01D). Os val ores obtidos durante os esforços foram enviados por uma interface ao computador e armazenado no programa para aquisição de dados Lab View na freqüência de $200 \mathrm{~Hz}$. Os dados obtidos foram submetidos ao processo de análise residual e suavizados utilizando o filtro "butterworth" de quarta ordem com freqüência de corte de $3 \mathrm{~Hz}$. Com a utilização da reta de calibração, os valores de tensão $(\mathrm{mV})$ foram convertidos em unidades de força ( $N$ ) pelo programa $M$ atlab 5.3, possibilitando assim a determinação das forças pico $\left(F P_{N A}\right)$ e média $\left(F M_{N A}\right)$ em nado atado.

O teste consistiu da aplicação de 1 esforço máximo de nado ondulatório com duração de $30 \mathrm{~s}$ e incentivo verbal dos atletas e pesquisadores. O início e o término do teste foram determinados por sinal sonoro (apito). Previamente ao início do esforço máximo os nadadores real izaram aproximadamente $10 \mathrm{~s}$ de nado moderado atados ao sistema de aquisição de dados. Durante os esforços, os nadadores estiveram com os braços estendidos sobre uma prancha comercial sendo solicitado aos atletas que mantivessem a cabeça fora da água.

Observou-se que os valores iniciais (aproximadamente $1 \mathrm{~s}$ ) foram bastante elevados, provavelmente devido aos esforços realizados durante a transição do nado moderado para o nado intenso. Desse modo os 200 pontos iniciais foram desconsiderados para não superestimarem os valores reais(15,19) (Figura 1).
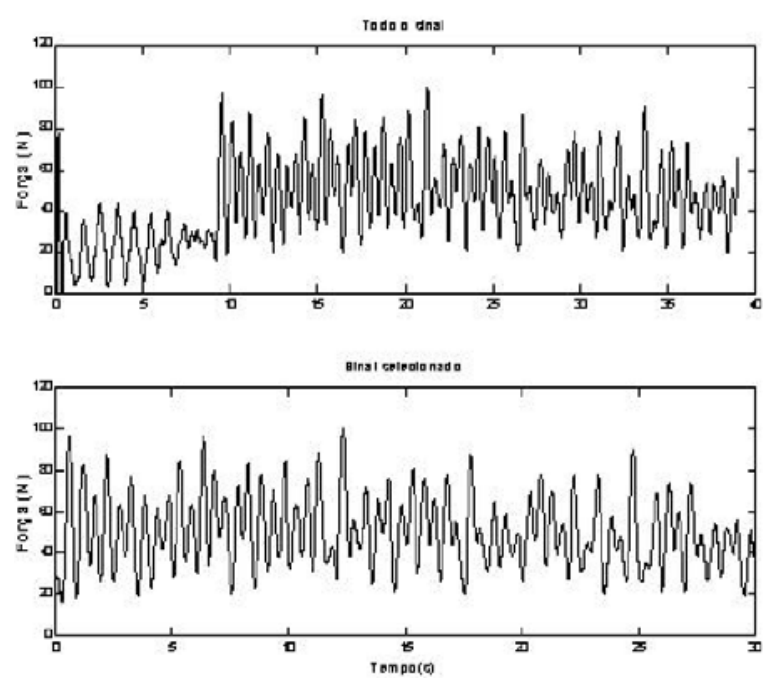

Figura 1. Comportamento de todo o sinal e somente o esforço máximo de um único nadador durante $30 \mathrm{~s}$ de teste de força dos membros inferiores de nado ondulatório em situação atada.

Tratamento estatístico

De acordo com o Shapiro-Wilk W test, o conjunto de dados apresentou distribuição normal; além disso, a homogeneidade foi confirmada através do Levene test. Dessa maneira, a comparação entre os valores obtidos no $\mathrm{NO}_{\mathrm{SB}}$ e $\mathrm{NO}_{\mathrm{SP}}$ utilizou-se o teste de Student para amostras dependentes. As possíveis relações entre os parâmetros obtidos a partir da $\mathrm{FMI}_{\mathrm{NO}}$ $\left(\mathrm{FP}_{\mathrm{NA}}, \mathrm{FM}_{\mathrm{NA}}\right)$ com os $\mathrm{NO}_{\mathrm{SB}}$ e $\mathrm{NO}_{\mathrm{SP}}$ foram analisadas através do teste de correlação de Pearson. Para todos os casos o nível de significância foi pré-fixado para $\mathrm{p}<0,05$.

\section{RESULTADOS}

Foram verificadas diferenças significativas entre a velocidade de nado obtida no $\mathrm{NO}_{\mathrm{SB}}\left(1,19 \pm 0,14 \mathrm{~m}^{\mathrm{s}} \mathrm{s}^{-1}\right)$ e NO $\mathrm{SP}_{\mathrm{SP}}\left(1,76 \pm 0,26 \mathrm{~m}^{-1}\right)$. No entanto esses valores foram altamente correlacionados $(r=0,92)$.

A $\mathrm{FM}_{\mathrm{NA}}$ correspondeu a $87 \%$ da $\mathrm{FP}_{\mathrm{NA}}$ (Figura 2). Tanto os valores de $\mathrm{FP}_{\mathrm{NA}}$ como os de $\mathrm{FM}_{\mathrm{NA}}$ apresentaram significativas correl ações com as velocidades dos $\mathrm{NO}_{\mathrm{SB}}$ e NO $\mathrm{NP}_{\mathrm{SP}}$. (Tabela 1). 


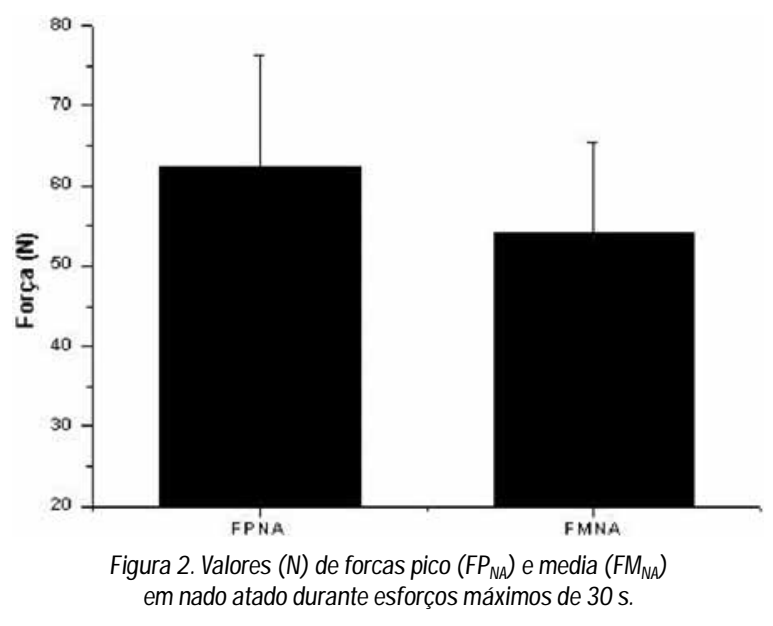

Tabela 1. Valores de correlação de pearson $(r)$ entre as forças pico $\left(F P_{N A}\right)$ e média $\left(\mathrm{FM}_{\mathrm{NA}}\right)$ em nado atado com as velocidades de nado ondulatório submerso $\left(\mathrm{NO}_{\mathrm{SB}}\right)$ e na superfície $\left(\mathrm{NO}_{\mathrm{SP}}\right)$.

\begin{tabular}{lcc}
\hline & $\mathrm{NO}_{\mathrm{SB}}$ & $\mathrm{NO}_{\mathrm{SP}}$ \\
\hline $\mathrm{FP}_{\mathrm{NA}}$ & $0,81 *$ & $0,80 *$ \\
$\mathrm{FM}_{\mathrm{NA}}$ & $0,80 *$ & $0,76 *$ \\
\hline & *Indica correlação significativa para $p<0,05$.
\end{tabular}

\section{DISCUSSÃO}

O principal achado do presente estudo foi a significativa correlação entre os valores de força de membros inferiores em nado ondulatório $\left(\mathrm{FMI}_{\mathrm{NO}}\right)$ com as velocidades de nado ondulatório na superfície $\left(\mathrm{NO}_{\mathrm{SP}}\right)$ e submerso $\left(\mathrm{NO}_{\mathrm{SB}}\right)$.

Considerando que variáveis como força e potência são parâmetros importantes na performance dos nadadores(18) e que a técnica de $\mathrm{NO}_{\mathrm{SB}}$ vem sendo amplamente utilizada, alem dos nados borboleta e costas, também no nado livre ${ }^{(3,9)}$, o presente estudo esteve empenhado em mensurar a $\mathrm{FMI}_{\mathrm{NO}}$ em situação de nado atado.

Yeater et al. (20) verificaram valores de $384 \pm 77 \mathrm{~N}$ e $693 \pm 231 \mathrm{~N}$ de força máxima durante as pernadas de crawl e peito respectivamente. Esses valores foram muito superiores aos verificados no presente estudo (força pico $=61,90 \pm 11 \mathrm{~N}$ ). No entanto esses pesquisadores não mensuraram a $\mathrm{FMI}_{\mathrm{NO}} \mathrm{e}$, apesar do ergômetro utilizado nesse estudo também conter células de carga como ao dinamômetro foram diferentes.
No estudo de Yeater et al.(20) os nadadores estiveram amarrados ao dinamômetro (fixado na plataforma de salto) por um fio de nylon. Este fio ainda passava por um sistema de polias localizado na borda oposta da piscina com relação à localização do dinamômetro. A parentemente esse sistema objetivou ajustar a tensão durante o processo de calibração. No presente estudo os nadadores foram amarrados ao dinamômetro que também esteve localizado na borda da piscina. No entanto foi utilizado um fio de aço com propriedade elástica reduzida(14,15,16) e a calibração desse sistema foi realizada com a adição de pesos conhecidos diretamente no dinamômetro. Com esse procedimento não é necessário que o fio de aço passe por um sistema de polias localizado na borda oposta da piscina.

É possível que as diferenças entre os ergômetros apresentada anteriormente, juntamente com as diferenças no nível dos nadadores bem como as particularidades de cada tipo de pernada tenham sido responsáveis pelas diferenças observadas nos valores de força. No presente estudo, os valores de correlação entre a $\mathrm{FP}_{\mathrm{NA}}$ e as $\mathrm{NO}_{\mathrm{SB}} \mathrm{eNO}_{\mathrm{SP}}$ foram superiores aos observados quando tendo a $\mathrm{FM}_{\mathrm{NA}}$ como variável dependente. Esses resultados contrariam os achados de Papoti et al. (16) que determinaram as $F_{N A}$ e $F P_{N A}$ e constaram que somente a $\mathrm{FM}_{\mathrm{NA}}$ apresentou correlações significativas com as performances de $100 \mathrm{~m}$ $(r=0,78), 200 \mathrm{~m}(0,82), 300 \mathrm{~m}(\mathrm{r}=0,75), 400 \mathrm{~m}$ $(0,74)$ e $600 \mathrm{~m}(r=0,70)$ nado crawl.

Essas contradições podem em parte ser explicada pela participação dos sistemas energéticos envolvidos. No estudo de Papoti et al.(16) os esforços variaram entre $100 \mathrm{~m}$ a $600 \mathrm{~m}$. Embora a predominância energética nos esforços prolongados seja aeróbia, pelo fato dos esforços terem sido supra limiares parte a energia necessária à realização do exercício provavelmente foi proveniente do sistema glicolítico enquanto que a energia necessária para a realização dos esforços de nado ondulatório em nado atado estaria mais relacionada com o sistema anaeróbio al ático.

Embora existam criticas quanto às corretas terminologias(7) as potências pico e média obtidas durante $o$ teste de Wingate estão associadas preferencialmente aos metabolismos anaeróbio al ático e lático respectivamente(1,17). Essa suposição justifica a maior corre- 
lação entre os valores de $F M_{\mathrm{NA}}$ e performances de $100 \mathrm{~m}$ a $600 \mathrm{~m}$ no estudo de Papoti et al.(16), enquanto que os esforços de nado ondulatório em nado atado estiveram, teoricamente, mais relacionados com a condição anaeróbia alática e, portanto, apresentaram maior correlação com a $\mathrm{FP}_{\mathrm{NA}}$. Interessante notar que a $\mathrm{NO}_{\mathrm{SB}}$ foi altamente correlacionada com a NO $\mathrm{NP}_{S \mathrm{P}}(\mathrm{r}=0,92)$. Esse achado sugere a possibilidade dos técnicos de natação utilizar também como elemento de treinamento exercícios compostos de $\mathrm{NO}_{\mathrm{SP}}$, mesmo quando o objetivo do treinamento for aprimorar a $\mathrm{NO}_{\mathrm{SB}}$. No entanto mais estudos são necessários para investigar especificamente a transferência dos efeitos do treinamento de $\mathrm{NO}_{S P}$ para a $\mathrm{NO}_{S \mathrm{BB}}$.

Durante os esforços de nado ondulatório em nado atado, três nadadores relataram a sensação de terem tocado os pés no fio de aço. Objetivando minimizar essa limitação potencial do ergômetro, a força pico foi assumida como a média dos valores de força durante os $5 \mathrm{~s}$ iniciais por ser o período nos quais os maiores val ores de potência no teste de Wingate são geralmente obtidos(6). No entanto, um período prévio de familiarização com o teste torna-se necessário para que os valores de força não sejam superestimados. Embora a mecânica de nado seja al terada durante o nado atado $(8,10)$ e os valores de força não reflitam necessariamente a força propulsiva gerada durante 0 nado livre(12), a determinação das $\mathrm{FP}_{\mathrm{NA}}$ eFM $\mathrm{NA}_{\mathrm{NA}}$ apresentaram-se adequadas para avaliação e predição das $\mathrm{NO}_{S P}$ e $\mathrm{NO}_{\mathrm{SB}}$ de nadadores treinado.

\section{CORRESPONDÊNCIA}

\section{Marcelo Papoti}

Endereço Departamento de Educação Física

IB, UNESP

Av. 24-A, 1515, Bela Vista

13506-900, Rio Claro - SP

Brasil

E-mail: mpapoti@yahoo.com.br 


\section{REFERÊNCIAS BIBLIOGRÁFICAS}

1. Bar-Or, O (1987). The Wingate Anaerobic Test: An Update on Methodology, Reliability and Validity. Sports M ed 4:381394.

2. Chollet-Tourny C, Chollet D, Hogie S, Papparodopoulos C (2002). Kinematic analysis of butterfly turns of international and national swimmers. J Sports Sci 20:383-390.

3. Clothier PJ, McElroy GK, Blanksby BA, Payne WR (2000). A comparison and analysis of traditional and modified tumble turns by age-group swimmers. J H um M ovement Stud 38:93-108.

4. Costill DL, Kovaleski J, Portes D, Kirwan J, Fieling R, King D (1985). Energy Expenditure During Front Crawl Swimming: Predicting Succes In Midle-Distance Events. Int J Sports M ed 6:266-270.

5. Hooper SL, Mackinon LT, Ginn EM (1998). Effects Of Three Tapering Techniques On The Performances Competitive Swimmers. Eur J A ppl Physiol 78:258-263.

6. Hutzler $Y$ (1998). Anaerobic fitness testing of wheelchair users. Sports M éd, 25:101-113.

7. Jacobs I, Bar-Or O, Karlsson J, Dotan R, Tesch P, Kaiser P, Inbar O (1982). Changes in muscular metabolites in females with 30-s exhaustive exercise. M ed Sci Sports Exerc 14: 457-460.

8. Lavoie JM, Leger LA, Leone M, Provencher PJ (1985). A maximal multistage swim to determine the functional and maximal aerobic power of competitive swimmers. J Swimming Res 1:17-22.

9. Lyttle AD, Blanksby BA, Elliott BC, Lloyd DG (2000). Net forces during tethered simulation of underwater streamlined gliding and kicking techniques of of the freestyle turn. J Sports Sci 18:801-807.

10. Maglischo CW, Maglischo EW, Sharp RL, Zier DJ, Katz A (1984). Tethered and nontethered crawl swimming. In: J. Terauds, K. Barthels, E. Kreighbaum, R. Mann, J. Crakes, C. A. Del Mar (Eds) Proceedings of ISBS: Sports Biomechanics 163-176.
11. Marinho PC, Andries OJr (2001). Avaliação da força propulsora do nadador: validação e reprodutibilidade de uma metodologia específica. Rev Brás Ciência e M ovimento (supl) 79.

12. Martin RB, Yeater RA, White MR (1981). A simple analytical model for the crawl stroke. Journal of Biomechanics 14:539-548.

13. Papoti M, Cunha SA, Martins LEB, Zagatto AM, Freitas PB, Gobatto CA (2005). Determinação da força e freqüência de braçada em nado atado utilizando sistema de aquisição de dados. In: XI Congresso Brasileiro de Biodinâmica 42.

14. Papoti M, M artins LE, Cunha SA, Zagatto A M, Gobatto CA (2007). Effects of taper on swimming force and swimmer performance after an experimental ten-week training program. J Strength Cond Res 21:538:542.

15. Papoti M, Martins LEB, Cunha SA, Zagatto AM, Gobatto CA (2003). Padronização de um protocolo específico para determinação da aptidão anaeróbia de nadadores utilizando células de carga. Rev Port Ciênc D esp 3:36-42.

16. Papoti M, Zagatto AM, Freitas PB, Cunha SA, Martins LEB, Gobatto CA (2005). Utilização do intercepto-Y na avaliação da aptidão anaeróbia e predição da performance de nadadores treinados. Rev Brás M éd Esporte 11 (2): 126130.

17. Smith JC, Hill DW (1991). Contribution of energy systems during a Wingate power test. Br J Sports M ed 25: 196-199.

18. Smith DJ, Norris RS, e Hogg MJ (2002). Performance Evaluation of Swimmers: Scientific Tools. Sports M ed 32;539-54.

19. Trappe S, Costill DL, Thomas, R (2001). Effect of swim taper on whole muscle and single muscle fiber contractile properties. M ed Sci Sports Exerc 33:48-56.

20. Yeater RA, Martin RB, White MK, Gilson HK (1981). Tethered swimming forces in the crawl, breast and beck strokes and their relationship to competitive performance. J Biomechanics 8:527-537. 